\title{
Phased Array Coil for Implementing Parallel MRI in Intravascular Imaging: A Feasibility Study
}

\author{
HAMMAD OMER, ${ }^{1,2}$ ROBERT J. DICKINSON, ${ }^{2}$ SHAKIL A. AWAN ${ }^{2,3}$ \\ ${ }^{1}$ Department of Electrical Engineering, COMSATS Institute of Information Technology, Islamabad, Pakistan \\ ${ }^{2}$ Department of Bioengineering, Imperial College London, United Kingdom \\ ${ }^{3}$ School of Mathematics and Computing, Faculty of Science and Environment, University of Plymouth, Drake Circus, \\ Plymouth, United Kingdom
}

\begin{abstract}
The use of intravascular MRI imaging of vessels will provide high quality images for improved diagnosis and treatment. An intravascular MR receive coil provides high SNR because of proximity to the tissue but respiratory and cardiovascular motion may affect the quality of acquired images. Parallel MRI provides a framework to reduce the scan time using multiple coils. This article presents a novel design of a Phased Array MR receive coil for intravascular imaging which can implement Parallel MRI. Cartesian and Radial GRAPPA are used as the main reconstruction algorithms. We demonstrate that Moving Segment Radial GRAPPA provides good image reconstruction with minimum artifacts when data is under-sampled by a factor of 16 . (c) 2015 Wiley Periodicals, Inc. Concepts Magn Reson Part A 00: 000-000, 2015.
\end{abstract}

KEY WORDS: parallel MRI; intravascular imaging; SENSE; GRAPPA; radial GRAPPA

\section{INTRODUCTION}

Magnetic Resonance Imaging (MRI) is a highly flexible imaging modality with many control parameters and provides robust soft tissue contrast. The ability of

Received 4 September 2013; revised 9 December 2014; accepted 26 February 2015

Correspondence to: Hammad Omer; E-mail: hammad_ar@hotmail. com

Concepts in Magnetic Resonance Part A, Vol. 00A(00) 00-00 (2015)

Published online in Wiley Online Library (wileyonlinelibrary. com). DOI: $10.1002 / \mathrm{cmr} . \mathrm{a} .21322$

(c) 2015 Wiley Periodicals, Inc.
MRI to obtain high resolution images can be utilized in intravascular imaging for the identification and characterization of vascular diseases e.g., atherosclerosis and may improve their treatment. A key component for intravascular imaging is a suitably designed receive coil that provides a high signal-to-noise ratio (SNR) when in close proximity to the anatomy of interest. The receive coil can also provide visual tracking of the catheter in real time without using any other imaging technique (e.g., fluoroscopy) thus increasing the accuracy of the diagnosis (1-3).

The feasibility of a catheter-based MRI was first shown by Kantorin 1984 (4) and since then many research groups have developed intravascular MRI 


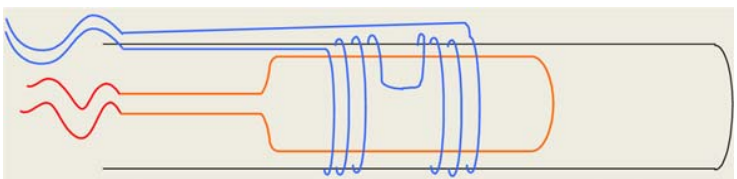

Figure 1 The schematic diagram of the proposed coil system.

coils $(5-10)$. However, respiratory and cardiovascular motion may badly affect the SNR and quality of the image in catheter based imaging (11). The reduction in MR scan time offered by parallel imaging may help in minimizing these motion artifacts.

Parallel MRI provides a framework to reduce the data acquisition time but it requires multiple coils for the data acquisition followed by a complex computation algorithm (e.g., SENSE, GRAPPA) (12). The spatial information inherent in each coil element is used to reconstruct the final image. This paper presents a novel design of a catheter based MR receiver coil system for intravascular imaging. This phased array coil is composed of two coil elements, a simple loop coil and an inverse solenoid coil. We have successfully implemented the phased array coil in parallel MRI and demonstrate that the scan time can be reduced by a factor of 16 using non-Cartesian techniques.

\section{THEORY}

The main function of an MRI receiver coil is to maximize the signal detection while minimizing the noise. An MRI receiver coil consists of a conductor, which is tuned and matched to receive the signals of only a particular frequency range. The proposed design of the MRI receiver coil system consists of two coil elements mounted on a catheter. One coil element is an inverse solenoid coil and the other is a simple loop coil. The coils are mounted on top of each other to achieve geometric decoupling (12) as shown in Fig. 1. Both coils are intrinsically decoupled because of the different designs and the sensitivity profiles of both coils are different (a fundamental requirement for the Parallel MRI). This particular phased array system is designed to support Parallel MRI and can accelerate the data acquisition process in intravascular imaging.

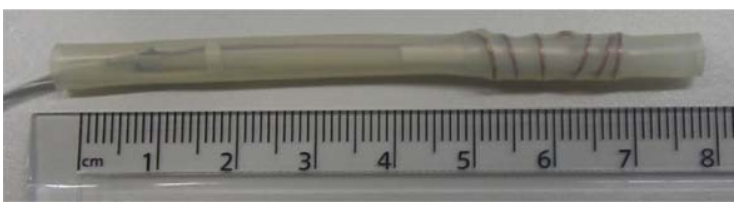

Figure 2 The final design of the receiver coil system.

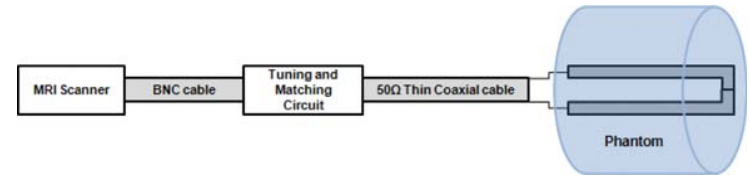

Figure 3 The model for the imaging experiments using MRI scanner.

Parallel MRI reconstruction algorithms can be classified into two main categories: (1) Image domain algorithms e.g., SENSE (13), (2) $k$-space algorithms e.g. GRAPPA (14). The discussion about these methods is beyond the scope of this paper but the details can be found in (12). However, the Parallel MRI methods which require the coil sensitivity information a priori may be difficult to implement in some situations e.g., in intra-vascular imaging when the imaging plane is continuously changing and the blood flow and respiratory movements (15) make it harder to acquire a good estimate of the receiver coil sensitivity. In the literature (16-18) there are suggestions for the use of $k$-space based Parallel MRI techniques [e.g., GRAPPA (14)] for intra-vascular MRI, where prior coil sensitivity information is not required to implement Parallel MRI (19-21).

GRAPPA (Generalized Auto-calibrating Partially Parallel Acquisitions) (14) is a $k$-space method for Parallel MRI image reconstruction where the linear combinations of the coil sensitivity variations of the receiver coil elements (in the receiver array) are used to estimate some of the gradient (or phase) encoding steps (14) thus allowing to skip some of the phase encoding steps in the MRI data acquisition. A weighted linear combination of the acquired points (source points) is used to generate the missing $k$-space points (the target points) (14), as shown by the following equation:

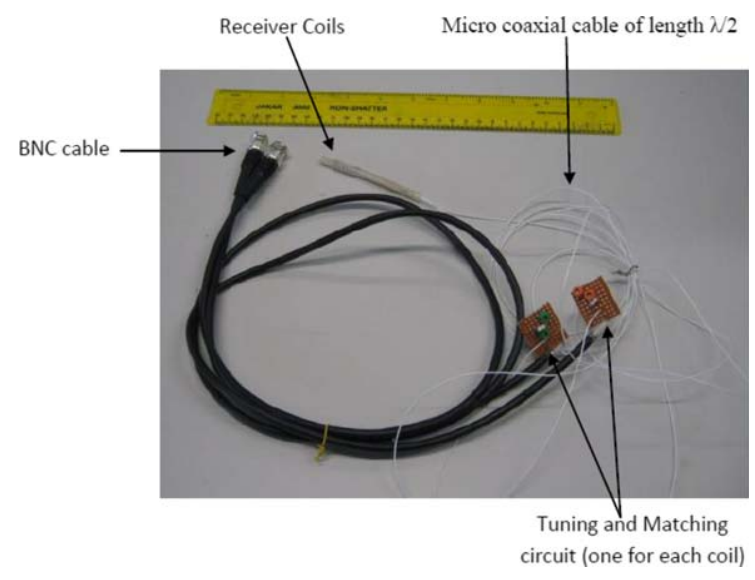

Figure 4 The phased array receiver coil system. 


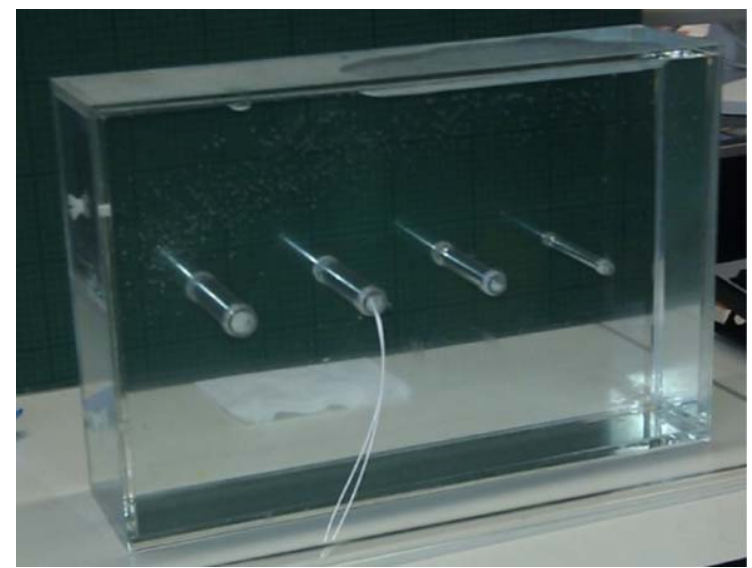

Figure 5 The designed uniform vascular phantom with tubes of four different diameters (outer diameters: $6 \mathrm{~mm}$, $8 \mathrm{~mm}, 10 \mathrm{~mm}, 12 \mathrm{~mm}$ ).
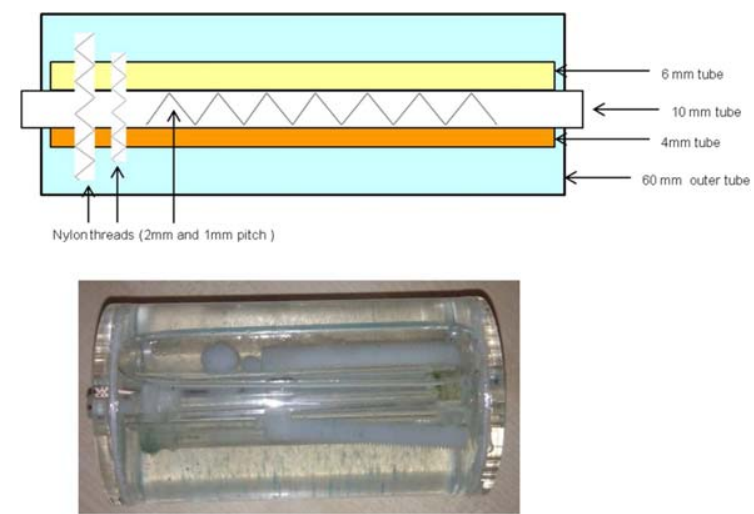

Figure 6 The vascular phantom with different geometric features, used to compare the quality of MR images using different reconstruction algorithms (Top) Schematic Diagram, (Bottom) Phantom.
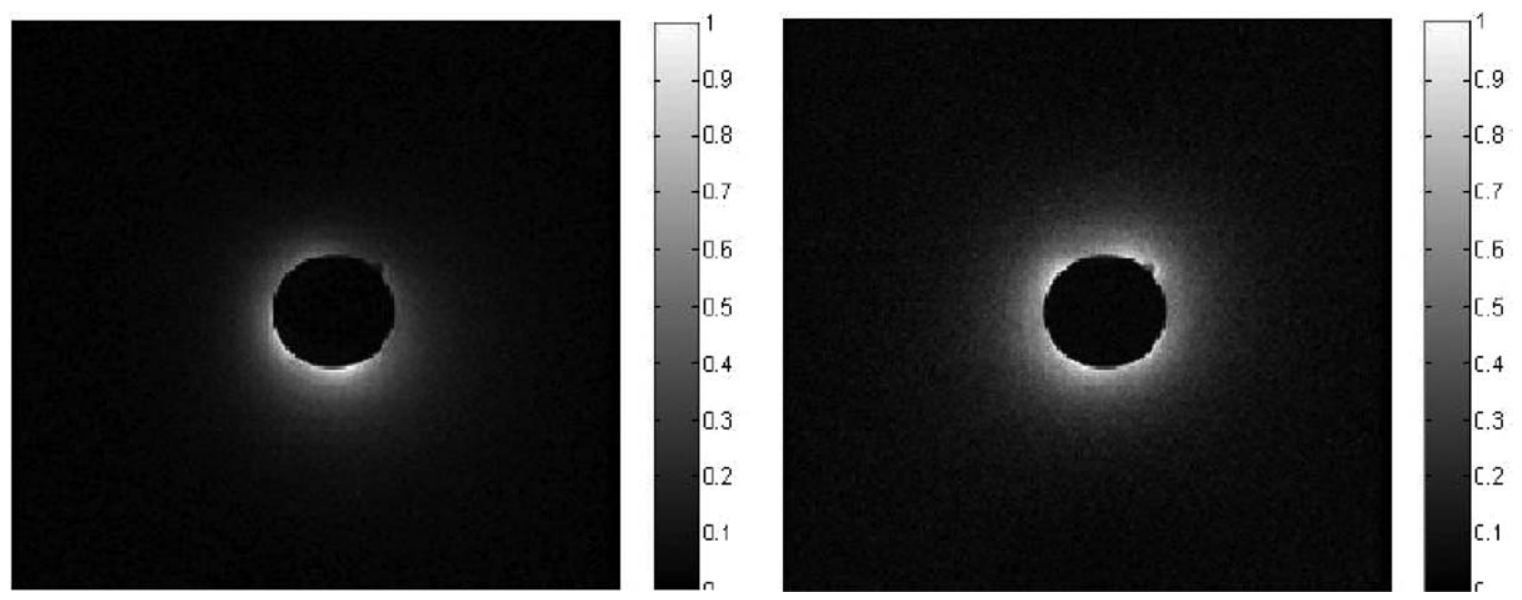

Figure 7 Comparison of the coil sensitivity in Axial direction $\left(T_{2}\right.$ weighted Fast Spin Echo sequence): (a) Simple Loop Coil (b) Inverse Solenoid Coil. 

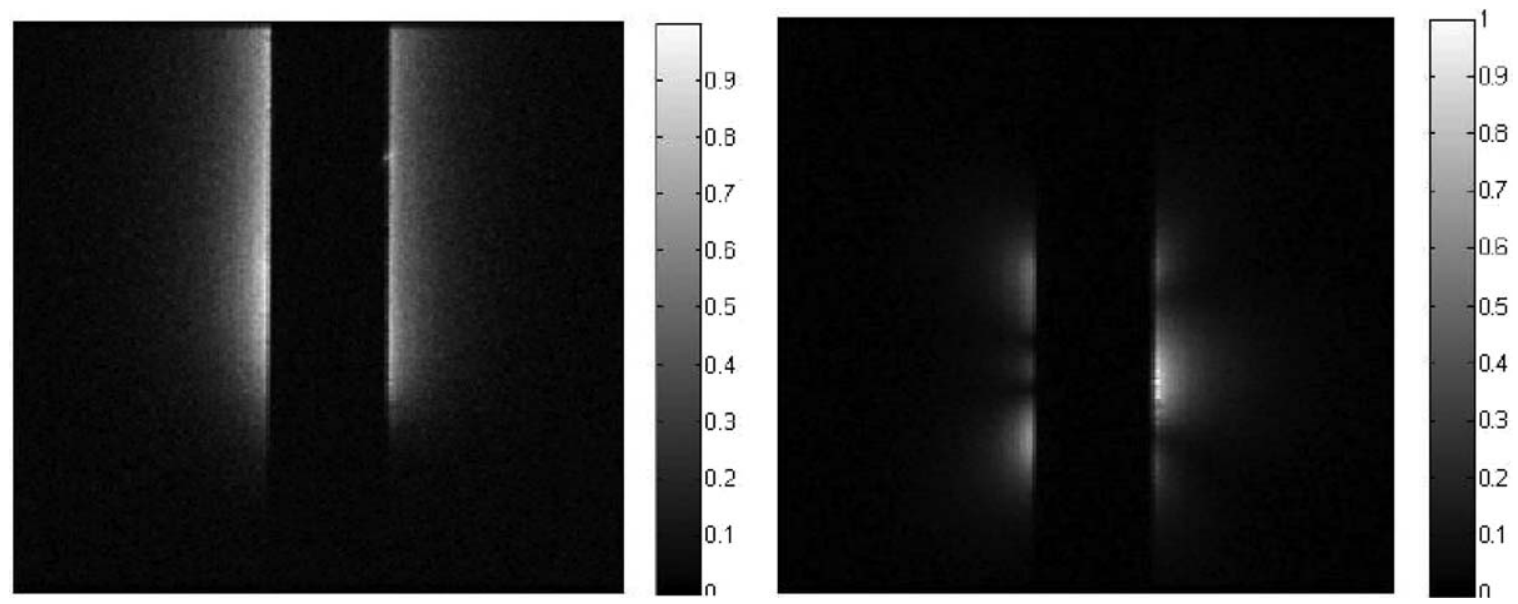

Figure 8 Comparison of the coil sensitivity in Coronal direction $\left(T_{2}\right.$ weighted Fast Spin Echo sequence): (a) Simple Loop Coil (b) Inverse Solenoid Coil.

$12 \mathrm{~mm}$ ) and can accommodate the receiver coils of varying diameters. The phantom has been filled with copper sulphate solution of $4 \mathrm{mM}$ concentration. This phantom is used to estimate the sensitivity profiles of the interventional receiver coils.

The second phantom is designed with some geometric features in it so that the quality of the reconstructed image (using different reconstruction algorithms) can be evaluated. The phantom is built in the form of a Perspex cylinder of $60 \mathrm{~mm}$ outer diameter. A tube of $10 \mathrm{~mm}$ diameter passes through the centre of the phantom (to hold the receiver coil) and protrudes out at both ends. Two Perspex tubes of different diameters $(4 \mathrm{~mm}$ and $6 \mathrm{~mm}$ ) are attached to the central $10 \mathrm{~mm}$ tube. The
$4 \mathrm{~mm}$ tube contains Copper Sulphate solution of $10 \mathrm{mM}$ concentration and is sealed at both ends, while the $6 \mathrm{~mm}$ tube contains the Copper Sulphate solution of $1 \mathrm{mM}$ concentration. The different concentrations of the solutions in the $4 \mathrm{~mm}$ and $6 \mathrm{~mm}$ tubes are aimed at providing different $T_{1}$ and $T_{2}$ relaxation times thus resulting in different contrasts in the MR images. Two nylon screws with $1 \mathrm{~mm}$ pitch and $2 \mathrm{~mm}$ pitch (M6 and M10, respectively) are also attached to the central $10 \mathrm{~mm}$ tube in the axial as well as longitudinal directions. These screws act as resolution bars and are placed to quantify the resolution capability of the different Parallel MRI reconstruction algorithms. The 60$\mathrm{mm}$ cylinder is filled with the $4 \mathrm{mM}$ Copper Sulphate

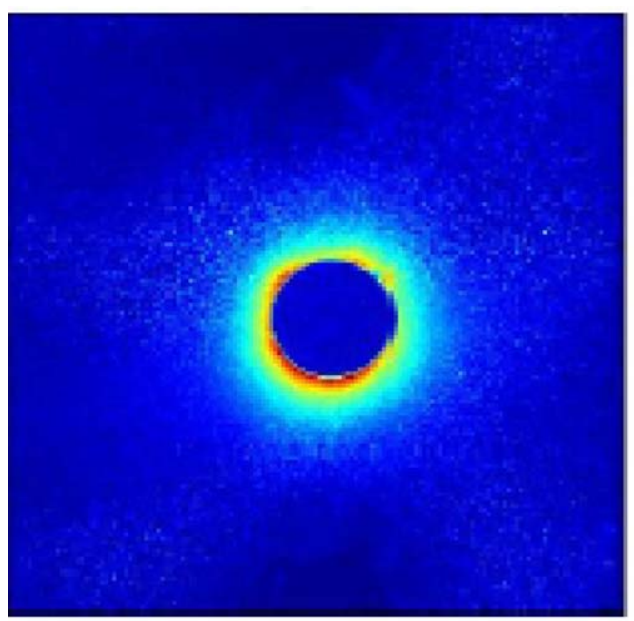

a
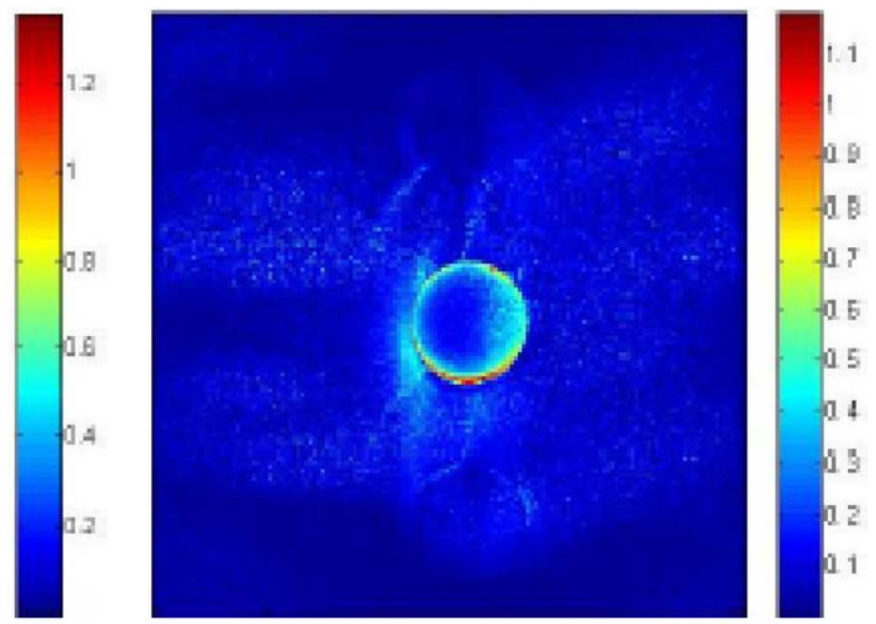

b

Figure 9 Cartesian GRAPPA reconstruction, each image obtained after the sum-of-squares reconstruction of the individually reconstructed coil images: (a) $\mathrm{AF} 2, \mathrm{AP}=0.069$, (b) $\mathrm{AF} 3$, $\mathrm{AP}=0.552$. 


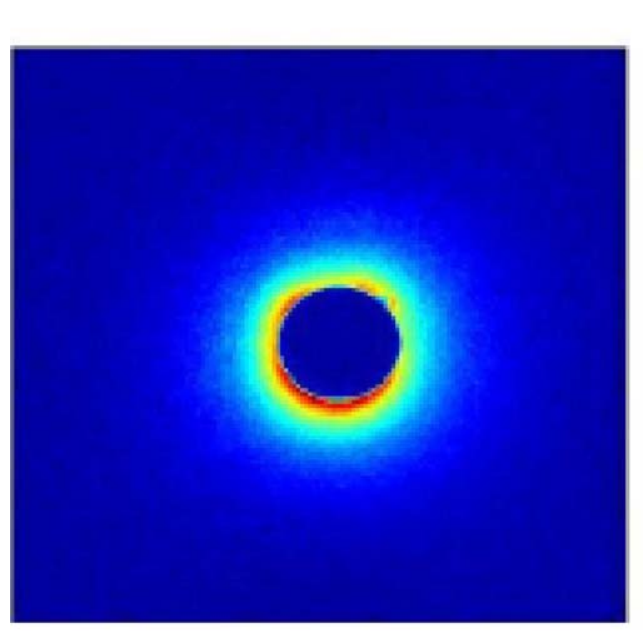

a
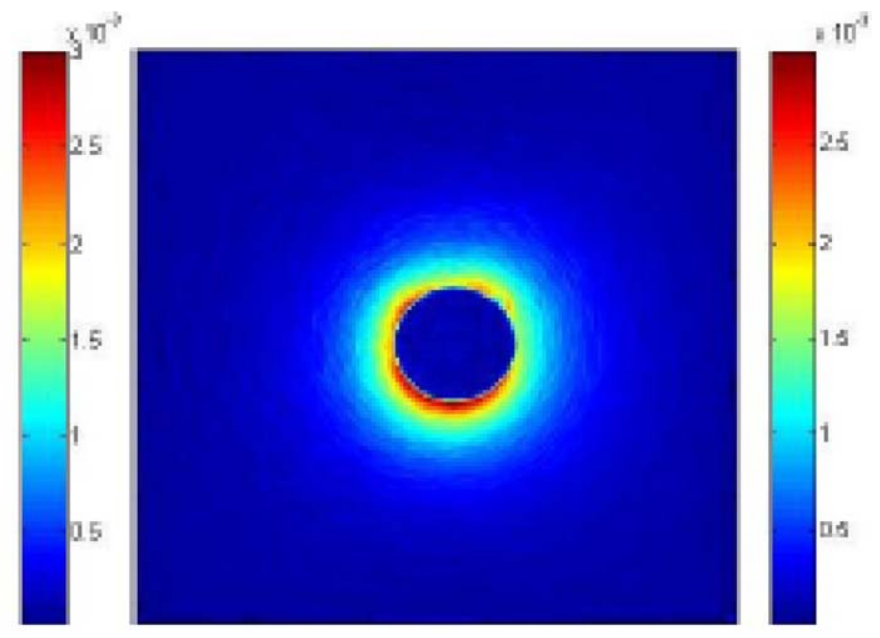

b

Figure 10 Standard Radial GRAPPA reconstructions for different acceleration factors (AF): (a) $\mathrm{AF}=2, \mathrm{AP}=0.014,(\mathrm{~b}) \mathrm{AF}=8, \mathrm{AP}=0.024$.

solution. The central $10-\mathrm{mm}$ tube along with all the associated structures is placed inside the $60-\mathrm{mm}$ cylinder and the system is sealed with Perspex glue. The phantom is shown in Fig 6.

The MRI data is acquired using GE MR750, 1.5T MRI scanner at St. Mary's Hospital London using Fast Spin Echo sequence with the following parameters: Slice Thickness $3 \mathrm{~mm}$, Matrix Size $256 \times 256$, Flip Angle $90^{\circ}$, TR $520 \mathrm{~ms}$, TE $15 \mathrm{~ms}$, FOV $55 \mathrm{~mm}$. First, a fully sampled dataset is acquired in Cartesian space and then converted to the Radial trajectory space using the modified version of the Matlab code provided by Nicole Seiberlich at ESMRMB Parallel MRI workshop in Wurzburg, Germany in 2010 (22).

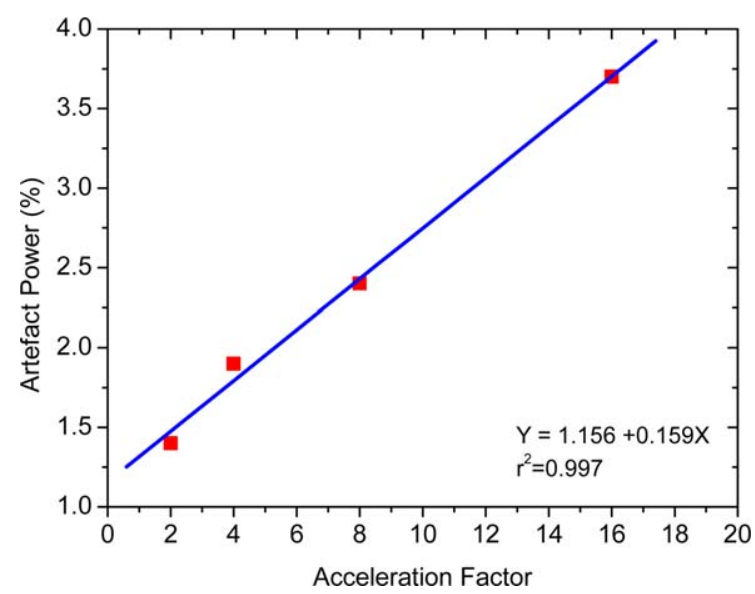

Figure 11 A plot of the artifact powers (\%) for different acceleration factors using Standard Radial GRAPPA.

\section{RESULTS}

In this paper Q-factor changes from 14 in the unloaded case to 13.69 in the loaded case for the simple loop coil, which is similar to a value reported by Homagk et al. (23). The inverse solenoid coil has a Q-factor of 15 in the unloaded case and 15.2 in the loaded case, indicating a good tuning and matching condition. The cross coupling S21 of the coil system is $-29 \mathrm{~dB}$ at 63.86 MHz (the tuning frequency for $1.5 \mathrm{~T}$ ) which indicates a good decoupling.

Figures 7 and 8 show the fully sampled axial and coronal slices through the uniform vascular phantom obtained using the intravascular phased array coil. The images show the difference between spatial sensitivity of both the coils which helps to achieve better reconstruction results in Parallel MRI (13).

Cartesian and Radial GRAPPA (12) reconstructions are performed for different acceleration factors. The reconstruction quality is evaluated using artifact power

Table 1 Artefact Power (AP) for Different Reconstruction Algorithms and Acceleration Factors (for Uniform Vascular Phantom Images)

\begin{tabular}{lccc}
\hline $\begin{array}{l}\text { Acceleration } \\
\text { Factor }\end{array}$ & $\begin{array}{c}\text { Cartesian } \\
\text { GRAPPA }\end{array}$ & $\begin{array}{c}\text { Radial } \\
\text { GRAPPA }\end{array}$ & $\begin{array}{c}\text { Radial } \\
\text { GRAPPA }\end{array}$ \\
\hline & & $\begin{array}{c}\text { (Still } \\
\text { Segment) }\end{array}$ & $\begin{array}{c}\text { (Moving } \\
\text { Segment) }\end{array}$ \\
\hline 2 & 0.069 & 0.014 & 0.009 \\
4 & - & 0.019 & 0.009 \\
8 & - & 0.024 & 0.0093
\end{tabular}




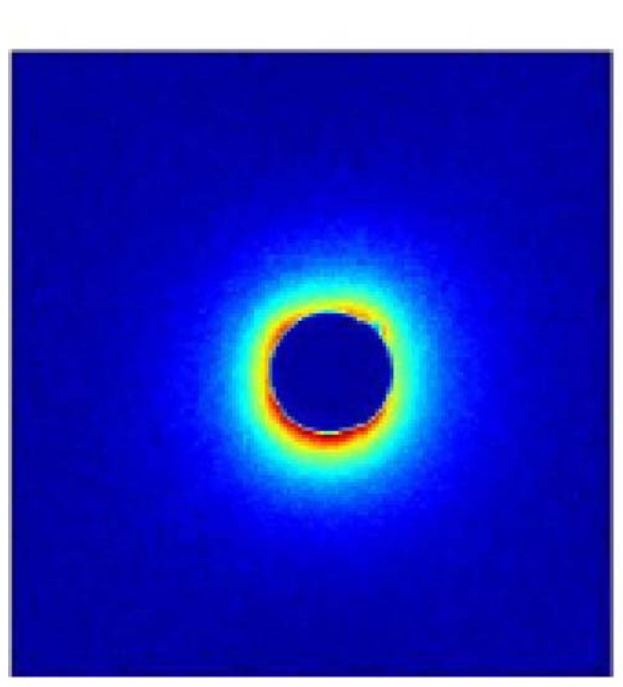

a

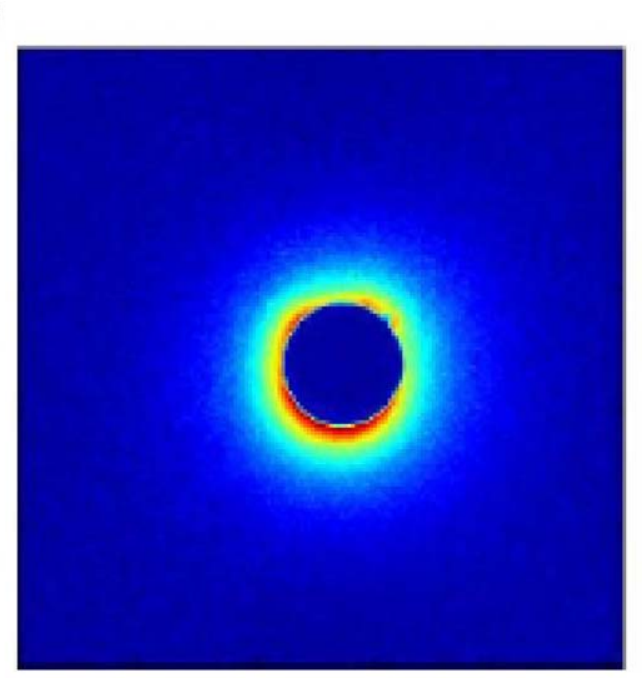

b
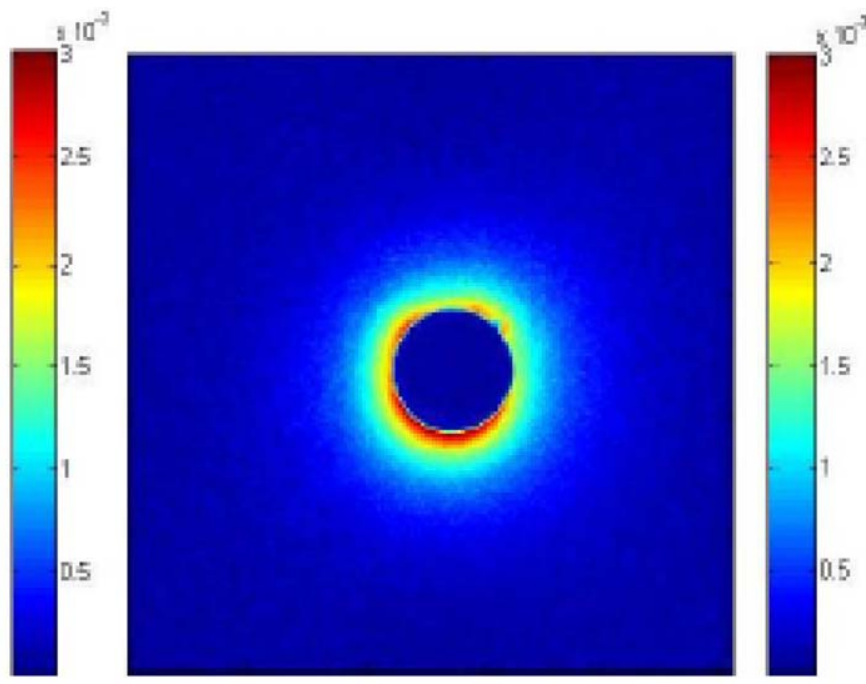

Figure 12 Moving Segment Radial GRAPPA reconstructions for different acceleration factors (AF): (a) $\mathrm{AF}=2, \mathrm{AP}=0.009$ (b) $\mathrm{AF}=8, \mathrm{AP}=0.0093$.
(AP). AP is a measure of accuracy and calculates the "Square Difference Error" between the reconstructed image and the reference image (24). Figure 9 shows the reconstruction results using Cartesian GRAPPA for acceleration factors 2 and 3 . The results indicate that Cartesian GRAPPA provides a good reconstruction for $\mathrm{AF}=2$ with a low AP of 0.069 ( $\sim 7 \%$ error $)$ but fails to provide a good reconstruction for $\mathrm{AF}=3$ (artifact power 0.552 ).

The reconstructed images using Standard Radial GRAPPA $(25,26)$ for different acceleration factors are shown in Fig. 10. A visual inspection of these images indicates the deteriorating quality of the reconstructed images at higher acceleration factors. Figure 11 shows a plot of the Artifact Power (AP) in

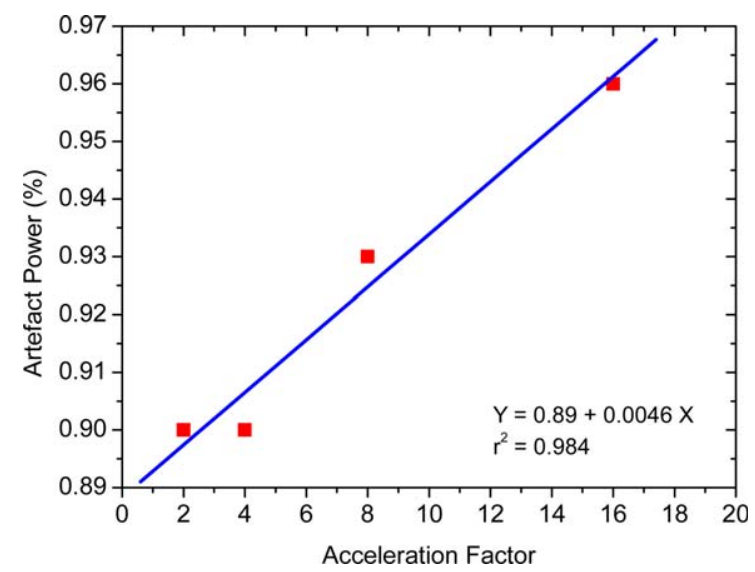

Figure 13 A plot of the artifact power vs. acceleration factors for Moving Segment Radial GRAPPA. the reconstructed images for the uniform vascular phantom using Standard Radial GRAPPA. As the acceleration factor increases, the artifact power increases as well, thus indicating a greater reconstruction error for higher acceleration factors e.g., the AP for acceleration factor 2 is only $1.4 \%$ but this increases to $3.7 \%$ for an acceleration factor 16 in this case (Table 1).

Figure 12 shows the reconstruction results for different acceleration factors using Moving Segment Radial GRAPPA $(25,26)$. The reconstructed images show a smaller artifact power even at higher acceleration factors. A plot of the AP vs. acceleration factors for Moving Segment Radial GRAPPA is shown in Fig. 13. The plot indicates an almost consistent reconstruction

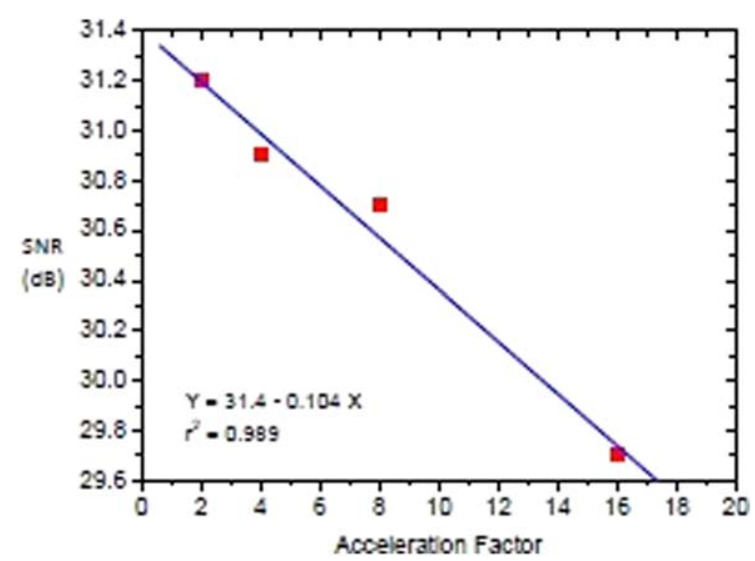

Figure 14 A plot of the SNR vs. acceleration factors using Moving Segment Radial GRAPPA. 

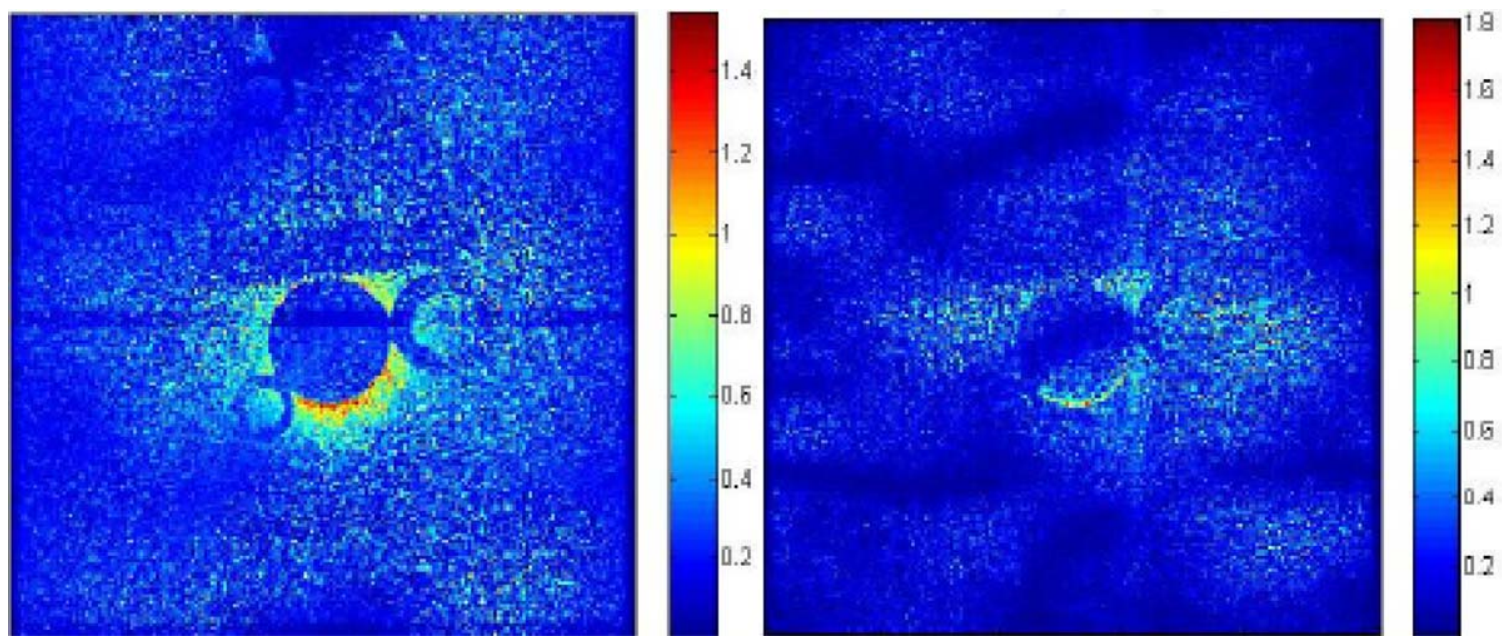

Figure 15 Cartesian GRAPPA reconstruction, each image obtained after the sum-of-squares reconstruction of the individually reconstructed coil images: (a) Acceleration Factor 2, $\mathrm{AP}=0.256,(\mathrm{~b})$ Acceleration Factor $3, \mathrm{AP}=0.439$.

quality with an AP of $0.9 \%$. There is a decrease in SNR in Moving Segment Radial GRAPPA as acceleration factor gets higher e.g., the SNR decreases by 5\% in this example when the AF changes from 2 to 16 in Moving Segment Radial GRAPPA and this is shown in Fig. 14.

Figure 15 shows the reconstruction performed for Cartesian GRAPPA using the under-sampled data of the geometric vascular phantom. Cartesian GRAPPA for $\mathrm{AF}=2$ provides apparently noisy reconstruction in this case with an error of $25.6 \%(\mathrm{AP}=0.256)$ but the reconstruction results are even worse for $\mathrm{AF}=3$ with $43.9 \%$ error $(\mathrm{AP}=0.439)$.

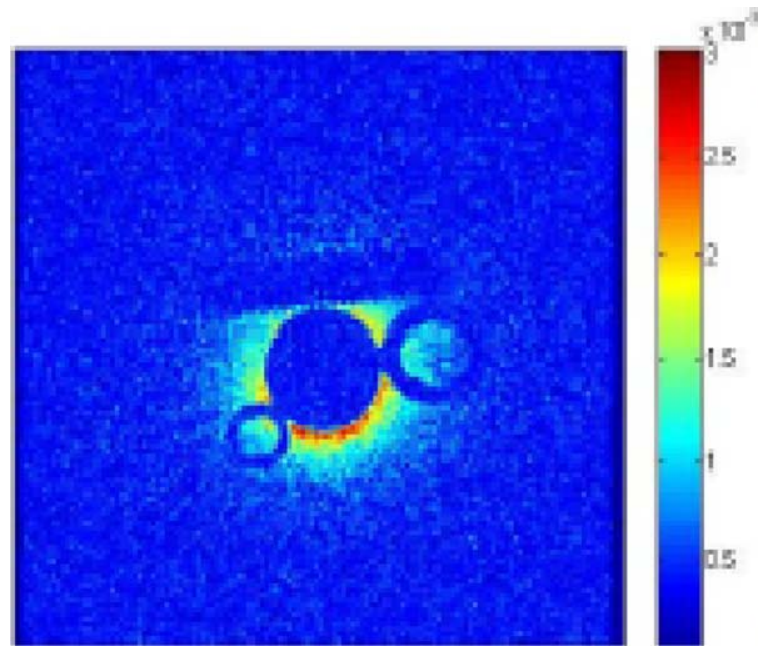

The reconstruction results using Moving Segment Radial GRAPPA for the geometric vascular phantom are shown in Fig. 16. The images show an error of only $2.26 \%$ and $2.32 \%$ for the acceleration factors of 2 and 8 , respectively. The $1 \mathrm{~mm}$ threads of the Nylon screw used as the resolution object are also visible in the reconstructed images.

\section{DISCUSSION}

The reconstruction results using Cartesian and Radial GRAPPA have been presented above in Section 4. The

Figure 16 Moving Segment Radial GRAPPA reconstructions for different acceleration factors, each image obtained after the sum-of-squares reconstruction of the individually reconstructed coil images: (a) $\mathrm{AF}=2, \mathrm{AP}=0.0226$ (b) $\mathrm{AF}=8, \mathrm{AP}=0.0232$. 
AP (24) is used to quantify the quality of reconstruction. Two objects (uniform vascular phantom and a geometric vascular phantom) are used in the imaging experiments. Cartesian GRAPPA seems to provide acceptable reconstruction results for acceleration Factor 2 but the reconstruction error (AP) becomes considerably higher for $\mathrm{AF}=3$ i.e., an error up to $43.9 \%$ (Fig. 9). It is important to note that the receiver coil system used in these experiments consists of only two coils (a simple loop coil and an inverse solenoid coil). The theoretical limit of acceleration for standard Cartesian reconstruction in Parallel MRI as described by Preussmann (13) is equal to the number of the coil elements in a parallel receiver array if each coil element has a distinct sensitivity profile (two coils in this case). Furthermore, the coil sensitivities shown here are different for both the coil elements as shown by the fully sampled images in Figs. 7 and 8 yet not necessarily fully independent. This demonstrates that the phased array coil can provide acceptable reconstruction results for $\mathrm{AF}=2$ but not for $\mathrm{AF}=3$.

The results also indicate that Radial GRAPPA helps in achieving higher acceleration factors as compared to Cartesian GRAPPA. This is because of an oversampling of the centre of $k$-space even at higher acceleration factors and the central part of the $k$-space contains most of the image information. However the Moving Segment Radial GRAPPA provides more consistent and better reconstruction results with minimum AP even at higher acceleration factors as compared with Standard Radial GRAPPA e.g., the maximum artifact power for Moving Segment Radial GRAPPA is only $2.32 \%$ for an acceleration Factor 8. In Moving Segment Radial GRAPPA a new segment is defined centred at each missing point in the under-sampled $k$-space and the corresponding weight set is applied to estimate the missing $k$-space signal (25). In this way, the segments here more accurately reflect the acceleration distance thus improving the quality of reconstruction as compared to Standard Radial GRAPPA where all the missing points in a segment are estimated using the same segment.

Moving Segment Radial GRAPPA is computationally expensive because a new segment is to be defined and weights to be calculated for each missing $k$-space point (26) in the under-sampled data. For a $256 \times 256$ image with an acceleration factor of 2, Standard Radial GRAPPA takes approximately 4 seconds on an Intel(R) Core $^{\mathrm{TM}} 2$ Duo, $2.66 \mathrm{GHz}$ system having $3.48 \mathrm{~GB}$ RAM, but Moving Segment Radial GRAPPA takes 24 s. Moving Segment Radial GRAPPA performs better but it is computationally more expensive (six times more expensive in this case) as compared to Standard Radial GRAPPA.
Some improvements in the design of the coils can improve the reconstruction results. The micro-coaxial cable connecting the tuning and matching circuit to the receiver coils may cause signal loss and thus limits the maximum achievable SNR. A better alternative will be to have the tuning and matching circuit integrated on the coil itself. This will minimize the signal losses and will improve the SNR. Although the current design of the coil system does not allow the capacitors to be placed on the coil or catheter, however, the use of miniature capacitors may help to achieve this in a future design.

One major safety concern regarding the use of active imaging catheters is the possibility of unwanted RF coupling which may lead to thermal injury owing to local RF heating effects $(27,28)$. Many techniques have been proposed to avoid RF induced heating $(29,30)$ and this aspect is beyond the scope of the current study. Future work involving clinical experiments may also require detailed investigation of the RF heating effects.

\section{CONCLUSIONS}

A new catheter-based phased array receiver coil is developed. The coil is composed of two coil elements (a simple loop coil and an inverse solenoid coil) and is capable of implementing Parallel MRI. The $Q$-factor calculations show good tuning and matching for both the coils in the unloaded as well as loaded conditions. The phased array MR receiver coil has been tested using different phantom objects. The imaging experiments are performed with 1.5 T GE scanner. Three different Parallel MRI algorithms (Cartesian GRAPPA, Standard Radial GRAPPA and Moving Segment Radial GRAPPA) are applied on the under-sampled data for different acceleration factors. The quality of the reconstructed images is quantified by AP. The results show that Moving Segment Radial GRAPPA provides better results with minimum artifacts as compared with the other two methods for the proposed phased array intravascular receiver coil.

\section{REFERENCES}

1. Ferrari VA, Wilensky RL. 2007. Intravascular magnetic resonance imaging. Topics Magn Reson Imaging 18:401-408.

2. Maehara A, Mintz GS, Weissman NJ. 2009. Advances in intravascular imaging. Circulation 2:482-490.

3. Doty FD, Entzminger G, Yang YA. 1998. Magnetism in high-resolution NMR probe design. I. general methods. Concepts Magn Resonance 10:133-156. 
4. Kantor HL, Briggs RW, Balaban RS. 1984. In vivo $31 \mathrm{P}$ nuclear magnetic resonance measurements in canine heart using a catheter-coil. Circulation Res 55: 261-266.

5. Hurst GC, Hua J, Duerk JL, Cohen AM. 1992. Intravascular (catheter) NMR receiver probe: preliminary design analysis and application to canine iliofemoral imaging. Mag Reson Med 24:343-357.

6. Martin AJ, Plewes DB, Henkelman RM. 1992. MR imaging of blood vessels with an intravascular coil. J Magn Reson Imaging 2:421-429.

7. Kandarpa K, Jakab P, Patz S, Schoen FJ, Jolesz FA. 1993. Prototype miniature endoluminal MR imaging catheter. J Vasc Interv Radiol 4:419-427.

8. Atalar E, Bottomley PA, Ocali O, Correia LC, Kelemen MD, Lima JA, Zerhouni EA. 1996. High resolution intravascular MRI and MRS by using a catheter receiver coil. Magn Reson Med 36:596-605.

9. Ocali O, Atalar E. 1997. Intravascular magnetic resonance imaging using a loopless catheter antenna. Magn Reson Med 37:112-118.

10. Quick HH, Ladd ME, Zimmermann-Paul GG, Erhart P, Hofmann E, von Schulthess GK, Debatin JF. 1999. Single-loop coil concepts for intravascular magnetic resonance imaging. Magn Reson Med 41:751-758.

11. Rivas PA, Nayak KS, Scott GC, McConnell MV, Kerr AB, Nishimura DG, Pauly JM, Hu BS. 2002. In vivo real-time intravascular MRI. J Cardiovasc Magn Reson 4:223-232.

12. Larkman DJ, Nunes RG. 2007. Parallel magnetic resonance imaging. Phys Med Biol 52:R15-R55.

13. Preussmann KP, Weiger M, Scheidegger MB, Boesiger P. 1999. SENSE: sensitivity encoding for fast MRI. Magn Reson Med 42:952-962.

14. Griswold MA, Jakob PM, Heidemann RM, Nittka M, Jellus V, Wang J, Kiefer B, Haase A. 2002. Generalized autocalibrating partially parallel acquisitions (GRAPPA). Magn Reson Med 47:1202-1210.

15. Awan SA, Mcginley JVM, Dickinson RJ, Angeli SI, Young IR. 2012. Design and development of a planar $\mathrm{B}_{0}$-coil for patient respiratory motion correction in magnetic resonance imaging. Concepts MR Part B 41:130-138.

16. Bock M, Muller S, Zuehlsdorff S, Speier P, Fink C, Hallscheidt P, Umathum R, Semmler W. 2006. Active catheter tracking using parallelMRI and realtime image reconstruction. Magn Reson Med55: 1454-1459.

17. Seiberlich N, Ehses P, Duerk J, Gilkeson R, Griswold M. 2011. Improved radial GRAPPA calibration for realtimefree-breathing cardiac imaging. Magn Reson Med 65:492-505.

18. Hillenbrand CM, Wong B, Griswold MA, et al. Intravascular parallel imaging: a feasibility study. Proc of 12th Annual Meeting of Intl Soc Mag Reson Med 2004, Kyoto, Japan, p 376.
19. Shin, PJ, et al. 2014. Calibrationless parallel imaging reconstruction based on structured low-rank matrix completion. Magn Reson Med 72:959-970.

20. Chen C, Li Y, Huang J. 2013. Calibrationless parallel MRI with joint total variation regularization. Medical Image Computing and Computer-Assisted InterventionMICCAI Springer-Berlin-Heidelberg, 8151:106-114.

21. Majumdar, A, Rabab, KW. 2012. Calibration-less multi-coil MR image reconstruction. Magn Resonance Imaging 30:1032-1045.

22. Seiberlich Nicole, Parallel Imaging Reconstruction II: Non-Cartesian, ESMRMB workshop on Parallel Imaging, Wurzburg, Germany, June 2010.

23. Homagk A-K, Umathum R, Korn M, Weber M-A, Hallscheidt P, Semmler W, Bock M. 2010. An expandable catheter loop coil for intravascular MRI in larger blood vessels. Magn Reson Med 63:517523.

24. Omer H, Dickinson R. 2010. A graphical generalized implementation of SENSE reconstruction using matlab. Concepts Magn Reson Part A 36:178-186.

25. Seiberlich N, Breuer F, Heidemann R, Blaimer M, Griswold M, Jakob P. 2008. Reconstruction of undersampled non-Cartesian data sets using pseudoCartesian GRAPPA in conjunction with GROG. Magn Reson Med 59:1127-1137.

26. Seiberlich N. 2010. Parallel imaging reconstruction. II. Non-Cartesian, ESMRMB workshop on Parallel Imaging, Wurzburg, Germany, June.

27. Konings MK, Bartels LW, Smits HFM, Bakker CJG. 2000. Heating around intravascular guidewires by resonating RF waves. J Magn Reson Imaging 12:79-85.

28. Nitz WR, Oppelt A, Renz W, Manke C, Lenhart M, Link J. 2001. On the heating of linear conductive structures as guide wires and catheters in interventional MRI. J Magn Reson Imaging 13:105-114.

29. Weiss S, Vernickel P, Schaeffter T, Schulz V, Gleich B. 2005. Transmissionline for improved RF safety of interventional devices. Magn Reson Med 54:182-189.

30. Krafft A, Muller S, Umathum R, Semmler W, Bock M. 2006. B1 field-insensitive transformers for RFsafe transmission lines. MAGMA 19:257-266.

\section{BIOGRAPHIES}

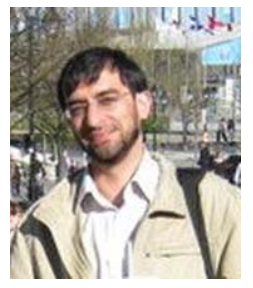

Dr Hammad Omer has been working as an Assistant Professor at Department of Electrical Engineering, COMSATS Institute of Information Technology (CIIT), Islamabad, Pakistan since March 2013. He is an Electronic Engineer with special interest in MRI (Magnetic Resonance Imaging). He received his $\mathrm{PhD}$ in MRI from Department of Bioengineering, Imperial College London in November 2012. He had been a Commonwealth scholar in UK between October 2008 and October 2012. During his $\mathrm{PhD}$ he has been involved in teaching MRI and other Medical Imaging Courses 
at Department of Bioengineering, Imperial College London. Earlier he did his MS in Bio-medical engineering (Medical Imaging stream) from Imperial College London in 2006, where he was honored with Bargarit Scholar of the year award for his outstanding performance during his MS. He has presented his research at many International conferences held in UK, Canada Germany and Sweden. He has also authored many research papers published in well-reputed international journals. He is a full member of International Society of Magnetic Resonance in Medicine (ISMRM), USA. Currently he is leading a research group of more than 35 researchers comprising $\mathrm{PhD}$, MS and BS students at Department of Electrical Engineering, CIIT, Islamabad, Pakistan.

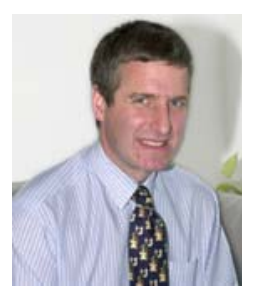

Dr Robert J. Dickinson M.A. PhD, MBA is a senior lecturer at the Department of Bio-engineering, Imperial College. He graduated with a degree in physics from Cambridge University, and then obtained a $\mathrm{PhD}$ in Biophysics from the University of London in ultrasound signal processing. Dr Dickinson has extensive experience in medical imaging, in both hospital and industrial environments. He worked on MRI coil development and system integration at Picker International Ltd and ultrasound imaging in a small start-up company where he developed a sub-1mm intravascular ultrasound imaging catheter for imaging coronary arteries. He has substantial experience in the bio-compatibility and other patient compatibility issues of invasive medical devices, together with commercialisation and IP transfer. He has filed over a number of patents and published papers, and has CE marked a number of medical devices. He has worked with Emcision Ltd on their range of electrosurgical devices with over 3000 patients treated to date. His current research interests include imaging in surgery, interventional imaging, miniaturising medical devices and electro surgery.

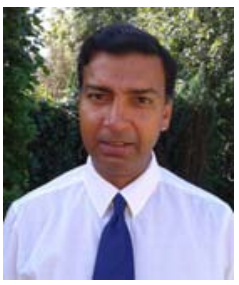

Shakil Awan is a Lecturer in Nanoelectronics at the University of Plymouth and an Academic visitor at the Cambridge Graphene Centre, University of Cambridge. Previously he was a Lecturer and Director of studies in Newnham College (2012-14) and a Senior Research Scientist at the UK's National Physical Laboratory (1997-2007). $\mathrm{He}$ has over 15 years of experience in precision electromagnetic measurements, covering superconductivity, electromagnetism, quantum Hall effect, MEMS, impedance standards and magnetic resonance imaging as well as more recently on graphene RF to THz electronics, plasmonics and biosensors. He was a Technical Programme Committee Member of CPEM 2008 as well as Chair of the special session on 'Nanoscale electromagnetic Metrology' at the conference. He has also served as a member of the Consultative Committee for Electricity and Magnetism (CCEM) Working Group on AC measurements of the quantized Hall resistance. His current research interests include graphene high-speed transistors, waveguides, mixers and biosensors. To date he has published 1 book and over 20 papers. 